\title{
Computational Challenges of Viscous Incompressible Flows
}

\author{
Dochan Kwak, Cetin Kiris and Chang Sung Kim \\ NAS Applications Branch, MS T27B-1 \\ NASA-Ames Research Center, Moffett Field, CA 94035
}

\begin{abstract}
Over the past thirty years, numerical methods and simulation tools for incompressible flows have been advanced as a subset of the computational fluid dynamics (CFD) discipline. Although incompressible flows are encountered in many areas of engineering, simulation of compressible flow has been the major driver for developing computational algorithms and tools. This is probably due to the rather stringent requirements for predicting aerodynamic performance characteristics of flight vehicles, while flow devices involving low-speed or incompressible flow could be reasonably well designed without resorting to accurate numerical simulations. As flow devices are required to be more sophisticated and highly efficient, CFD tools become increasingly important in fluid engineering for incompressible and low-speed flow. This paper reviews some of the successes made possible by advances in computational technologies during the same period, and discusses some of the current challenges faced in computing incompressible flows.
\end{abstract}

\section{INTRODUCTION}

Almost all, if not all, fluid is compressible. Incompressible flow is an approximation of flow where flow speed is insignificant everywhere compared to the speed of sound of the medium. If incompressible flow is defined in this way, the majority of the fluid and associated flow we encounter in our daily lives belong to the incompressible category. For example, most of the flow associated with air and water (such as flow related to automobiles, ships, submarines, the water supply through pipes and channels, hydraulic turbines, pumps, lowspeed airplanes, and trout swimming in mountain streams) and flow of biofluid (such as blood) are all in the incompressible flow domain. One of the earliest mathematical models of incompressible flow is the famous equation by Bernoulli, who in 1730 developed the model equation while investigating blood flow (see an interesting article by Quarteroni [1]). It is not surprising that scientists have been investigating incompressible flow analytically, experimentally, and computationally ever since.

Computational study of incompressible flow problems in both basic research and engineering applications has been performed for several decades. Numerical solutions for such basic fluid dynamics problems as: flow past a circular cylinder; flow through channels, ducts and pipes; and flow over a backward facing step were presented as early as the 1930s (e.g., Thom [2] for a circular cylinder). In the computational fluid dynamics (CFD) community, especially in aerospace, CFD is synonymous with computational aerodynamics. Computational incompressible fluid dynamics was treated as a special case of aerodynamics, that is, the low-speed limit of compressible fluid dynamics. This is probably due to requirements to produce highly accurate results for predicting aerodynamic performance characteristics of flight vehicles, while flow devices involving low-speed flow can be reasonably well designed without resorting to accurate numerical simulations (e.g., hydraulic turbines for hydroelectric power plants were designed without the CFD approach). As flow devices become increasingly compact and efficient-pushing the conventional operating envelope-requirements on incompressible CFD tools have become more demanding, just as aerodynamic performance prediction tools require quantitative prediction capability. This trend is reflected in the development of various incompressible flow solution methods and tools, especially, in conjunction with high-fidelity computations using high-end computing facilities.

Mathematically, the incompressible flow formulation poses unique issues not present in compressible equations 
because of the incompressibility requirement. Physically, information travels at infinite speed in an incompressible medium, which imposes stringent requirements on computational algorithms for satisfying incompressibility as well as difficulties in designing downstream boundary conditions. Differences in various methods of solving the incompressible flow equations originate from differences in strategies of satisfying incompressibility.

Generally, two different approaches are used in solving viscous incompressible flow equations, i.e., incompressible Navier-Stokes equations. The first is based on satisfying the incompressibility directly. If primitive variables, i.e., pressure and velocity, are chosen, pressure is used as a mapping parameter to satisfy incompressibility. This class of methods is generally known as the "pressure-based" method. Instead of pressure and velocity, derived quantities such as stream function-vorticity and vorticity-velocity can be used, resulting in different sets of governing equations. For general three-dimensional applications, however, the primitive variable formulation poses the least difficulty in geometry modeling and in setting the boundary conditions. The second approach is based on compressible flow formulation where momentum and continuity equations are coupled through the use of density. Incompressibility is recovered as a limiting case of this formulation. This class of methods is known as the "density-based" method. The artificial compressibility method is typical of this approach, which is a special case of preconditioned compressible flow methods.

During the past several decades, many review articles and books on CFD have discussed incompressible flow methods. For more comprehensive reviews of computational methods for incompressible flow in general, readers are referred to these materials, i.e.: Roach [3]; Peyret and Taylor [4]; Hirsch [5]; Kwak [6]; Gunzburger and Nicolades [7]; Hafez and Oshima [8]; Gresho and Sani [9]; and Hafez [10]. These books and articles provide fairly extensive formulations, numerical methods, and solutions to fundamental fluid dynamic problems. This paper gives a short summary of methods from a historical perspective. After thirty-some years of numerical simulation of incompressible flows, industrial problems involving complex systems are now solved routinely. This paper focuses on engineering applications to review what has been accomplished to date and what challenges remain to be resolved. There are a vast number of cases where incompressible CFD methods have made significant impacts. Those presented here represent limited samples to illustrate the level of complexities researchers encounter in fluid engineering.

\section{EVOLUTION OF SOLUTION METHODS}

For general applications, the approach based on primitive variables is the most flexible choice. Other formulations using derived variables, such as stream function and vorticity, eliminate the need for pressure boundary conditions. However, this comes at the expense of adding a new requirement for derived variables, such as vorticity boundary conditions. All these formulations produce comparable results for two-dimensional problems, however, extension to three dimensions is not straightforward for stream function formulation. For vorticity-velocity formulation in three dimensions, three Poisson-type vorticity equations are introduced that make the formulation very expensive to solve. In this paper, the examples of three-dimensional applications have primarily been obtained using primitive variable formulation. Therefore, this section briefly reviews the evolution of the two most popular approaches using the primitive variables. Other formulations and examples are found in the literature [3-10].

\subsection{Formulation}

Three-dimensional incompressible flow with constant density is governed by the following Navier-Stokes equations:

$$
\begin{aligned}
& \frac{\partial u_{i}}{\partial x_{i}}=0 \\
& \frac{\partial u_{i}}{\partial t}+\frac{\partial u_{i} u_{j}}{\partial x_{j}}=-\frac{\partial p}{\partial x_{i}}+\frac{\partial \tau_{i j}}{\partial x_{j}}
\end{aligned}
$$


Where $t$ is the time, $x_{i}$ the Cartesian coordinates, $u_{i}$ the corresponding velocity components, $p$ the pressure, and $\tau_{i j}$ the viscous stress tensor. Here, all variables are non-dimensionalized by a reference velocity and length scale. For turbulent flow, $\tau_{i j}$ should include turbulent stresses.

The major difference between the incompressible and the compressible Navier-Stokes formulations is in the continuity equation. The incompressible formulation can be viewed as a singular limit of the compressible one. Satisfying the mass conservation equation, therefore, is the primary issue in solving the above set of equations. Physically, incompressible flow is characterized by elliptic behavior of the pressure waves, the speed of which in a truly incompressible flow is infinite. The pressure field is desired as a part of the solution; however, the pressure condition is needed on the boundary for numerical computation. One can utilize other formulations using derived quantities like vorticity to eliminate the pressure from the boundary conditions; however, boundary conditions for the derived variables have to be imposed. In realistic 3-D problems, these derived quantities are difficult to define or impractical to use. The primitive'variable formulation, namely, using pressure and velocities as dependent variables, then becomes very convenient and flexible in 3-D applications. However, in this formulation, mass conservation and its relation to pressure must be properly handled while achieving computational efficiency. Although various techniques have been developed in the past, none have proven to be universally better than the others.

\subsection{Methods Based on Pressure Iteration}

In 1965, Harlow and Welch [11] published the first primitive variable method using a derived Poisson equation for pressure. In this method, called the marker-and-cell (MAC) method, the pressure is used as a mapping parameter to satisfy the continuity equation. By taking the divergence of the momentum equation, the Poisson equation for pressure is obtained:

$$
\nabla^{2} p=\frac{\partial h_{i}}{\partial x_{i}}-\frac{\partial}{\partial t} \frac{\partial u_{i}}{\partial x_{i}}=g
$$

Where

$$
h_{i}=-\frac{\partial u_{i} u_{j}}{\partial x_{j}}+\frac{\partial \tau_{i j}}{\partial x_{j}}
$$

The usual computational procedure involves choosing the pressure field at the current time step such that continuity is satisfied at the next time step. The original MAC method is based on a staggered arrangement on a 2-D Cartesian grid. The staggered grid conserves mass, momentum, and kinetic energy in a natural way and avoids odd-even point decoupling of the pressure encountered in a regular grid [12]. However, these properties become unclear when applied to generalized curvilinear coordinates. Even though the original method used an explicit Euler solver, various time-advancing schemes can be implemented here. Ever since its introduction, numerous variations of the MAC method have been devised and successful computations have been made.

The major drawback of this method is the large amount of computing time required for solving the Poisson equation for pressure. When the physical problem requires a very small time step, the penalty paid for an iterative solution procedure for the pressure may be tolerable. But the method as a whole is slow and the pressure boundary condition is difficult to specify. One important aspect of the numerical solution of the Poisson equation for pressure is tied to the spatial differencing of the second derivatives. To satisfy the mass conservation in grid space, the difference form of the second derivative in the Poisson equation has to be constructed consistently with the discretized momentum equation (see Kwak [6] for more detail).

For a steady-state solution, the correct pressure field is desired only when the solution is converged, so the iteration procedure for the pressure can be simplified such that it requires only a few iterations at each time step. The best-known method using this approach is the Semi-Implicit Method for Pressure-Linked Equations (SIMPLE) [13-14]. The unique feature of this method is the simple way of estimating the velocity correction and thus the pressure correction. This feature simplifies the computation but introduces empiricism into the 
method. Despite its empiricism, the SIMPLE method has been used successfully for many computations, and has been the basis for many pressure-based methods developed since (e.g., [15] and many others). It is not, however, the intention of this paper to fully review the evolution of this method. Many successful applications and tools developed to date are the real testimonial of the value of this pressure-based approach, in general.

\section{Fractional-step procedure for pressure-based method}

The MAC method can be viewed as a special case of the projection method (i.e., Chorin, [16]) or, from the operator point of a view, one variation of the fractional-step method [17-19]). Here, operator splitting can be accomplished in several ways by treating the momentum equation as a combination of convection, pressure, and viscous terms. The common application of this method is done in two steps: The first step is to solve for an auxiliary velocity field using the momentum equation, in which the pressure-gradient term can be computed from the pressure in the previous time step or can be excluded entirely. In the second step, the pressure is computed, which can map the auxiliary velocity onto a divergence-free velocity field. This procedure was first applied in staggered Cartesian grids by Harlow and Welch [11], and later extended to regular grids (e.g., Dwyer et al. [20] and many others).

One particular aspect of the fractional-step method requiring special care is the intermediate boundary condition. Orszag et al. [21] discussed this extensively. Rosenfeld et al. [22] devised a generalized scheme where physical boundary conditions can be used at intermediate steps. As with other pressure-based methods, the efficiency of the fractional-step method depends on the Poisson solver. A multi-grid acceleration, which is physically consistent with the elliptic field, is one possible avenue to enhance the computational efficiency. In light of successfil computations in Cartesian coordinates using staggered grid, it became of interest to develop a method for general three-dimensional coordinates. However, extension of the staggered arrangement to general computational cells is not straightforward and involves features related to geometric conservation. A general numerical method based on a staggered grid was developed at NASA Ames Research Center (among others), initially by Rosenfeld et al. [22]. Later, Kiris and Kwak [23] developed a more robust implicit procedure for "not so smooth grids" using a finite-volume framework on a staggered grid. A staggered grid has favorable properties in Cartesian coordinates, such as coupling odd-even points. However, it is debatable whether a staggered grid has clear advantages over a regular grid in generalized curvilinear coordinates. In practice, this is because flux balancing in general curved cells requires interpolation that makes the differences between a cell-centerted and staggered arrangement insigniñcant. Further discussion reiated to this approach is found in recent collection of papers by Hafez [10] (for example, in papers by Loner et al. [24] and more).

\subsection{Artificial Compressibility Method}

Advances in CFD for aerospace have been made in conjunction with compressible flow computations. Therefore, it is of significant interest to be able to use some of these compressible flow algorithms. One of the early attempts to do this was the artificial compressibility method by Chorin (1967) [25]. In this formulation, the continuity equation is modified by adding a time-derivative of the pressure term resulting in:

$$
\frac{1}{\beta} \frac{\partial p}{\partial t}+\frac{\partial u_{i}}{\partial x_{i}}=0
$$

where $\beta$ is an artificial compressibility or a pseudo-compressibility parameter. These two terms, artificial compressibility and pseudo-compressibility, are used interchangeably in the literature. Together with the unsteady momentum equations, this forms a hyperbolic-parabolic type of time-dependent system of equations. Thus, the implicit schemes developed for compressible flows can be implemented. Note that $t$ no longer represents a true physical time in this formulation.

Physically, this means that waves of finite speed are introduced into the incompressible flow field as a medium to distribute the pressure. For a truly incompressible flow, the wave speed is infinite, whereas the speed of propagation of these pseudo-waves depends on the magnitude of the artificial compressibility. Additionally, in a true incompressible flow, the pressure field is affected instantaneously by any disturbances in the flow field, but with artificial compressibility, there is a time lag between the flow disturbance and its effect on the pressure field. Ideally, the value of the artificial compressibility is chosen to be as high as the particular choice of 
algorithm allows, so that the incompressibility is recovered quickly. This must be done without decreasing the accuracy and the stability property of the numerical method implemented. On the other hand, if the artificial compressibility is chosen such that these waves travel too slowly, then the variation of the pressure field accompanying these waves will be very slow. This will interfere with the timely development of the viscous boundary layer. In viscous flows, the behavior of the boundary layer is very sensitive to the streamwise pressure gradient, especially when the boundary layer is separated. If separation is present, a pressure wave traveling with finite speed will cause a change in the local pressure gradient, which will affect the location of the flow separation. This change in separated flow will feed back to the pressure field, possibly preventing convergence to a steady state. Especially for internal flow, the viscous effect is important for the entire flow field and the interaction between the pseudo pressure-waves and the viscous flow field becomes very important.

Artificial compressibility relaxes the strict requirement to satisfy mass conservation in each step. However, to utilize this convenient feature, it is essential to understand the nature of artificial compressibility both physically and mathematically. Chang and Kwak (1984) [26] reported details of artificial compressibility, and suggested some useful guidelines for choosing the artificial compressibility parameter.

For obtaining steady state solutions, the governing equations are iterated in pseudo-time until the solution converges to steady state, at which time the original incompressible Navier-Stokes equations are satisfied. Time-dependent calculation of incompressible flows are especially time consuming due to the elliptic nature of the governing equations. Physically, this means that any local change in the flow has to be propagated to the entire flow field. Numerically, this means that in each time step, the pressure field has to go through one complete steady-state iteration cycle; for example, by a Poisson-solver-type pressure iteration or an artificial compressibility iteration method. In transient flow, the physical time step has to be small and, consequently, the change in the flow field may be small. In this situation, the number of iterations in each time step for obtaining a divergence-free flow field may not be as high as for regular steady-state computations. However, timeaccurate computations are in general extremely time-consuming. Therefore, it is particularly desirable to develop computationally efficient methods by implementing a fast algorithm and by utilizing computer characteristics such as parallel processing.

Various applications that evolved from the artificial compressibility concept have been reported for obtaining steady-state solutions (e.g., Kwak et al. [27], Chang et al. [28], Choi and Merkle [29]). To obtain timedependent solutions using this method, an iterative procedure can be applied in each physical time step such that the continuity equation is satisfied. Merkle and Athavale [30] and Rogers et al. [31] reported successful computations using this pseudo-time iteration approach.

\section{Pre-conditioning}

In incompressible flow, the density is constant; this is the source of difficulty in solving the compressible Navier-Stokes equation as the flow approaches the incompressible limit. Adding a non-physical pressure term [25] made it possible to adopt a time-like iteration scheme for solving the governing equations. The success of this approach prompted a series of attempts to develop preconditioning methods to be used in conjunction with compressible Navier-Stokes equations (Turkel [32], Van Leer et al. [33], Choi and Merkle [34], Venkataswaran and Merkle [35]). This method is especially valuable for solving aerodynamic flow fields involving multiple scales (i.e., flow fields involving regions of very low speed in generally high-speed flow problems). This formulation continues to be utilized in simulating multi-phase flow such as cavitation in liquid flow [36].

\section{Numerical Schemes}

It is common to see that the artificial compressibility code resembles that of a compressible flow. For example, to alleviate the difficulty of solving an unfactored implicit scheme, indirect methods have been devised by many researchers. The alternating direction implicit (ADI) scheme by Beam and Warming [37] or Briley and McDonald [38] approximates the implicit operator in the unfactored scheme by a product of three one-dimensional operators. This scheme is unconditionally stable in two dimensions. In three dimensions, with the use of numerical dissipation, this scheme becomes conditionally stable. The ADI scheme introduces a factorization error that reduces the rate of convergence. This method introduced perhaps the first implicit scheme to CFD, thus viscous flow computation became more feasible. With the help of reduced cost by the diagonalization of the Jacobian 
(Pulliam and Chaussee [39]), this scheme has been used in the development of many flow solvers. At NASA Ames, INS3D, the first incompressible Navier-Stokes solver in a generalized three-dimensional coordinate, (Kwak et al. [27]), was developed using this scheme. The original INS3D code was extensively used as a primary design tool for an upgraded design of the Space Shutile main engine (SSME) hot gas manifold by Chang et al. [28]. This was probably the first major CFD contribution to rocket propulsion system design.

In the artificial compressible formulation, since the governing equations are changed into the hyperbolicparabolic type, some of the upwind differencing schemes developed for the compressible Euler and NavierStokes equations [40-43] can be used. These schemes were implemented into the flow solver successfully using the artificial compressibility method by Rogers et al. [31]. A variety of iterative schemes can be implemented in conjunction with the upwind schemes. For example, Rogers et al. [31] solved the resulting set of numerical equations using an unfactored line relaxation scheme similar to that employed by MacCormack [44].

\subsection{Other Methods}

Although the evolution of incompressible flow solver development has been explained here using examples the authors of this paper are directly involved with, a large number of research projects have been ongoing in the incompressible CFD community in general. In particular, development along the finite element or unstructured grid approach has not been examined in this paper. Readers interested in this area are referred to a fairly recent book by Gresho and Sani [9], and a number of articles in the book by Hafez [10] (for example, see articles by Glowinski [45], Morgan [46], Tezduyar [47], as well as a many articles covering numerical methods and special topics). Several other methods related to incompressible flow computations can be found in the references cited.

In general it is the authors' opinion that there are no longer any major differences in the basic methodologies and tools used by different organizations. Differences in robustness, computational efficiencies, and accuracy usually come from how the solution procedures are set up in conjunction with geometry and grid handling, software engineering, and some subtle algorithmic aspects not well-suited to presentation in written papers.

\section{Computed Examples}

\section{Solutions to some basic problems}

Validation computations of solution algorithms and flow solvers have been reported in the literature over the years; e.g., problems like square cavity, flow over a circular cylinder, duct flow of various shapes, backward facing steps, juncture flow, and tip and wake vortex flow. Even though the geometry of these flow problems are relatively simple, they offer rich sources for studying flow physics and opportunities to prove the validity of particular numerical methods and procedures. One simple example is shown in Figure 1, where the artificial compressibility and pressure projection methods are compared in order to evaluate the capability for predicting unsteady phenomena. Once the final solutions are obtained, the two approaches can produce equivalent results, however, the iterative processes for the artificial compressibility method and time advancing scheme for the pressure projection scheme have to be properly handled [48].

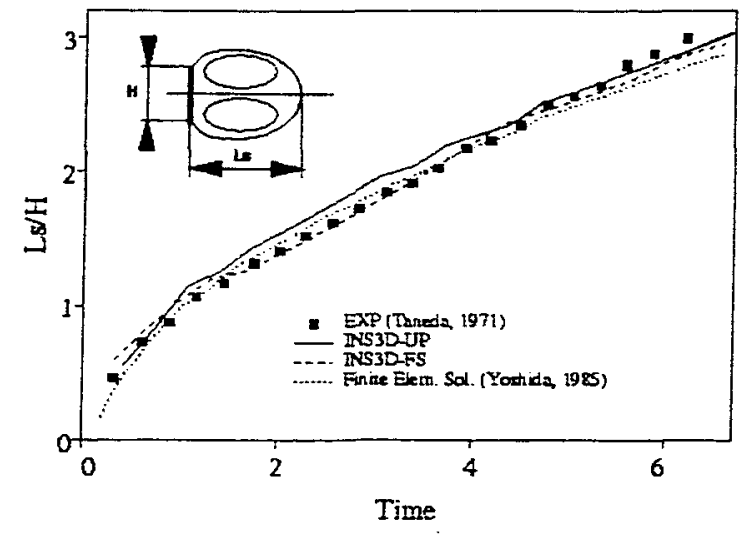


Figure 1. Evolution of the stagnation point for an impulsively started flat plate: Comparison of artificial compressibility (denoted by INS3D-UP), projection method (INS3D-FS), finite-element solution [49], and water tank experiment [50].

\section{Validation for real-world applications}

Validation of the solution procedure for real-world applications takes another level of effort because the flow field involving complex geometry often requires high resolution locally to resolve the complicated flow, as well as requiring evaluation of physical model being implemented. Such a validation effort is of significant importance to problems like submarines with propulsor and for turbopump subsystems for liquid rocket engines.

Figure 2 illustrates one such validation using a pump impeller. This validation is the first step toward further application of the simulation procedure to an entire turbopump system. Figure 2a shows an impeller geometry with surface grids. This particular configuration was designed to collect experimental data for developing an advanced impeller. Figure $2 b$ shows some comparison of computed results with test data. Even with this simplified geometry, testing, which involves variations in flow conditions and shroud geometry, is not straightforward. Simulation involves geometric modeling and grid generation, as well as approximations related to numerical methods and physical modeling. As shown in the figures, the computed results are not on top of test data - unlike the basic flow problems cited above. In engineering applications, especially for preliminary design applications, one needs to determine the acceptable level of accuracy. More importantly, the numerical simulation procedure must produce consistent results when flow conditions are varied as well as for variations in geometry such as different angle, length, and lean of impeller blades.

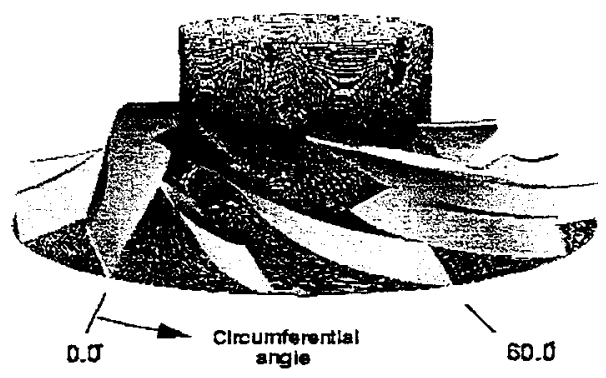

Figure 2a. Geometry of a test impeller.

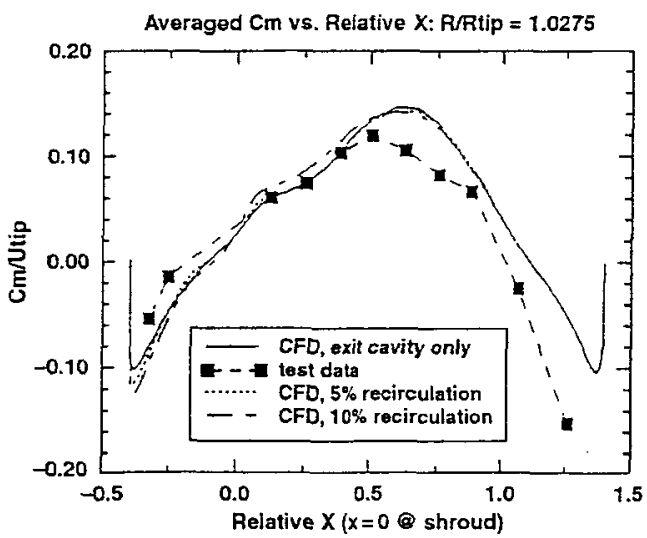

Figure $2 \mathrm{~b}$. Comparison of circumferentially averaged meridional velocity at the impeller exit.

\section{Applications to large engineering problems}


Once validated, the computational procedure can then be extended to solve engineering problems. One of the most significant applications of incompressible flow simulation began in the early 1980s, when NASA carried out a series of upgrades to the Space Shuttle main engine (SSME) developed in the 1970s. One such effort was the powerhead redesign. Considered the backbone of the engine, the powerhead consists of the main injector assembly and pre-bumers. Partially burned hor gas passes through Hot Gas Manifold (HGM) to the main injector assembly. From 1983-84, the powerhead redesign was undertaken, focusing on a two-duct HGM. NASA Ames and Rocketdyne collaborated in applying the INS3D code to this task. Since the Mach number of the partially burned gas was very low, the incompressible approximation was a valid assumption. The team of researchers successfully applied a CFD simulation procedure to this task for enhancing the performance of the SSME powerhead. The new design was developed based on a two-duct hot gas manifold. The two-duct design replaced the previous three-duct engine, smoothing the fuel flow, reducing pressure and turbulence, and lowering temperatures in the engine during operation. This two-duct design first flew on the shuttle in July 1995. It significantly imnroved fluid flow in the system, thus reducing maintenance and enhancing overall engine performance. This pioneering work was probably the first major application of incompressible CFD to a rocket propulsion system (see [28] for more detail).

Since this work, many incompressible flow software packages have been developed for analyzing complex engineering systems. One such example is the UNCLE code [36], which has been applied to various naval hydrodynamic applications. Many other equally significant software packages exist, as well. It is not the purpose of this paper to list the inventory of such codes.

A typical process of flow simulation, especially, for high-fidelity unsteady flow, requires large amounts of both

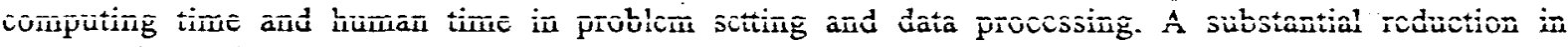
computational time for 3-D unsteady flow simulations is desired to reduce the design-cycle time of, for example, a pump system. Part of this speedup will be due to enhancements in computer hardware. The remaining portion of the speedup must be contributed by advances in grid-generation procedures, flow solution algorithms, and by efficient parallel implementations. In the following example, an overset strategy [51] was adopted to generate the grid for multiple components of a pump. To reduce human and computing time for grid generation, scripting [52] has been developed that substantially reduce the grid-generation portion of the total simulation time. Figure 3 illustrates this procedure.

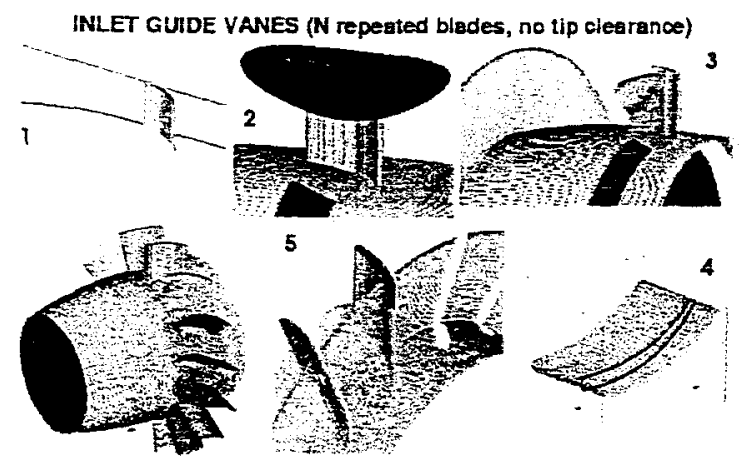

Figure 3. Illustration of automated grid-generation procedure with turbopump scripts for inlet guide vanes.

Parallel computing strategies vary depending on computer architecture such as memory arrangement relative to processing units. Two approaches are illustrated here: the first approach is hybrid MPIOpenMP and the second is Multi-Level Parallelism (MLP), developed at NASA Ames. The former is obtained by using message-passing interface (MPI) for inter-zone parallelism, and by using OpenMP directives for intra-zone parallelism. INS3DMPI is based on the explicit message-passing interface across MPI groups and is designed for coarse-grained parallelism. 


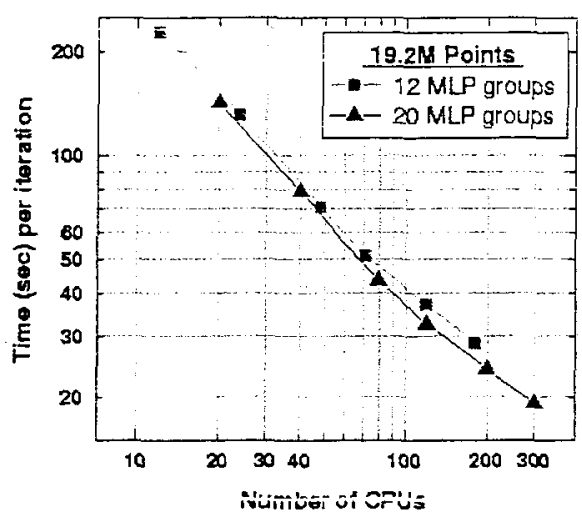

Figure 4. Parallel performance of INS3D-MLP on SGI Origin platform.

The second approach to parallel computing is obtained by using NAS-MLP routines. This approach differs from the MPI/OpenMP approach in a fundamental way in that it does not use messaging at all. All data communication at the coarsest and finest level of parallelism is accomplished via direct memory referencing instructions. This approach also provides a simpler mechanism for converting legacy code than does MPI. For shared memory MLP, the coarsest level parallelism is supplied by spawning independent processes via the standard UNIX fork. The boundary data for the overset grid system is archived in the shared memory arena by each process. Other processes access the data from the arena as needed. Figure 4 shows the MLP scalability for impeller computations using 19.2 million grid points.

\section{Biomedical / Biofluid Applications}

Extension of incompressible flow methods to blood flow has been of interest to biomedical researchers for many years. For the cardiovascular system, the brain, and other parts of the human body, branching of blood vessels involves bifurcations, most of which are non-symmetric. Therefore, bifurcation has been a popular subject for blood flow simulations. Bifurcation problems offer the opportunity to address numerical issues involving grid generation, as well as to study basic fluid dynamic phenomena relevant to blood circulation simulation. When the size of a blood vessel becomes very small (as in capillaries), non-Newtonian characteristics become significant, thus requiring physical modeling. In addition, the vessel wall is elastic, requiring a structural model to account for geometric changes, depending on wall stresses. Early pioneering work on this subject was done by Womersley [53]. More sophisticated computations have since been performed for regular and non-regular bifurcating arteries including stenotic vessels (see Berger [54] for a review).

The human circulatory system is like a huge tree with many branches of various sizes. Therefore, many computational studies have been performed using a truncated geometric model. One difficulty of simulating a truncated arterial system results from setting proper boundary conditions, especially at the downstream boundary. To account for the large arterial network, Quarteroni and his colleagues have developed a circuit analog, which has been applied to more inclusive circulatory systems modeling such as the cardiovascular system and the Circle of Willis in the brain (see [55] among many publications). An alternative way of imposing downstream boundary conditions has been developed by Tim David and his colleagues [56] in their computation of the Circle of Willis (CoW).

One crucial step in biomedical flow simulation is to construct computational geometry starting from anatomical data. Kim et al. reconstructed a 3-D, anatomically realistic CoW geometry from human-specific magnetic resonance angiography (MRA) provided by researchers at Canterbury University in New Zealand (Tim David, Mechanical Engineering Dept; John Fink and Mike Hurrell, Christchurch Hospital). The image segmentation procedure is illustrated in Figure 5. The raw MR images were converted to the RGB graphic file format. After extracting the segments of interest by filtering voxels with intensities below a certain threshold, a segmentoutlining algorithm was used to display the extracted objects on each sectional layer. 


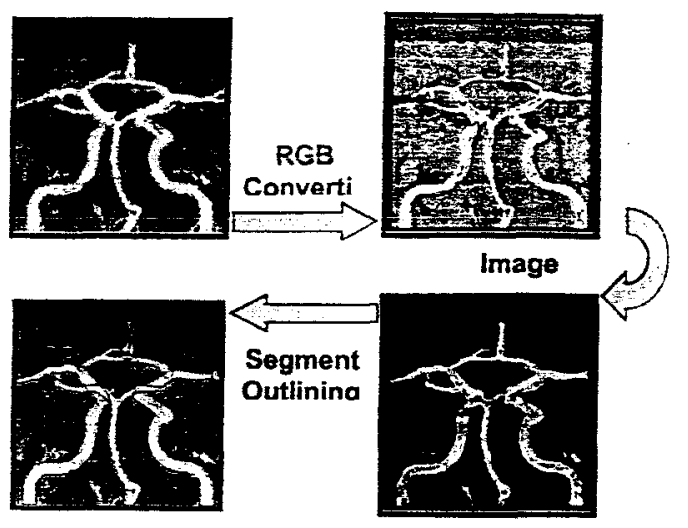

Figure 5. Image segmentation from a magnetic resonance image for a human-specific Circle of Willis. (MRA provided by John Fink and Mike Hurrell, Christchurch Hospital, New Zealand.)

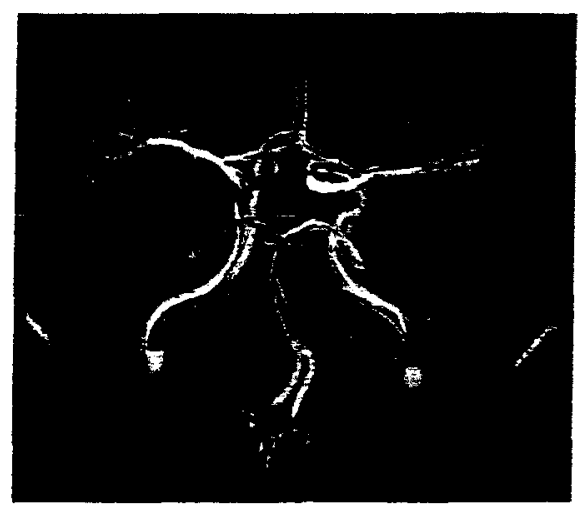

Figure 6. Overlapping of a pulsatile blood flow simulation at peak systole on a magnetic resonance angiogram.

With a non-Newtonian blood model, a model for deformable blood vessels, truncated downstream boundary conditions, and an auto-regulation model, Kim et al. [57] simulated unsteady blood circulation through this CoW under various gravitational conditions (a snapshot is shown in Figure 6, above).

Modeling and simulation of the cardiovascular system has been the subject of many investigations because of its importance in biomedical research. Peter Hunter and his colleagues have been modeling cardiovascular systems, including multidisciplinary aspects, producing one of the most impressive results to date (e.g., see [58]). Earlier and perhaps the most elaborate physical model of the heart was pioneered by Peskin and his colleagues (see [59] for example) since 1970s. This model included blood, wall structures, and electrical field activating heart muscles.

Another interesting application of blood flow simulation is related to artificial devices such as artificial hearts, ventricular assist devices (VADs), and heart valves. Because the demand for transplant organs far exceeds the number of donors, the need for artificial devices-to be used either as a temporary device or as a permanent replacement for a natural organ-becomes increasingly high. Accurate quantification of blood flow plays a crucial role in developing these devices. Thus, CFD simulation of blood flow in and around these artificial devices has become an indispensable part of the design. One such example is the recent CFD-aided design of the DeBakey VAD, which enabled human implantation by removing thrombus formation and lowering hemolysis to an acceptable level for human application. This effort set a new milestone for CFD application to the biomedical area [60].

All such blood flow computations may be regarded as a branch of incompressible CFD. The number of CFD applications to blood flow and biomedical problems are increasing rapidly, and the work cited here represents only small samples of the vast amount of ongoing and existing work.

\section{Challenges}

Incompressible CFD methodologies have been advanced along with computational technologies in general. Many fluid engineering problems can now be simulated, however, mostly at a single-component level. For example, it is possible to generate solutions to problems like a turbopump (inducer-impeller-diffuser), a naval vehicle at a steady motion including propulsor by a model, and a truncated model of the brain or heart. To realize the full benefits of CFD, more inclusive modeling will be required, such as, systems of pumps including multiples of pumps and feed lines, vehicle in maneuver with propulsor, a complete or more inclusive human circulatory system from the heart through the aorta and all the way to the brain and kidney capillaries. Attempts to solve these types of problems have been made with some qualitative successes. However, the predictive capability is still very limited and prediction with accurate physics is yet be accomplished. This will require inclusion of not only fluid dynamic modeling but modeling of other quantities like thermal loading, structural 
properties (such as structural behavior of arterial walls), and cavitation physics. These computations will require not only large computing resources but large data storage and management technologies, as well.

Flow solver codes and software tools have been developed to the point that many daily fluid engineering problems can now be computed routinely. Some of the physical models, such as those for turbulence and transition, however, have not advanced much since the '70s or ' $80 \mathrm{~s}$. Other models, like the cavitation model, have yet to be advanced to produce quantitative results for engineering. In aerospace design, the most productive aspect of CFD applications has been to predict relative change among design variations. To push the limit of operation and to try bold new ideas, more predictive capabilities will be needed for complicated flow's involving transient phenomena, separation, tip vortex, and cavitation. For example, without accurate prediction capabilities for such quantities as cavitation and damaging frequencies, back flow, and rotational stall, CFD can't be of much help in the development of an advance turbopump system. To make these advances, highfidelity computation using high-end computing facilities are still be a "must"- despite the current euphoria about the PC clusters and grid computing.

One final note is related to human resources. Even though CFD has advanced remarkably, many challenging cases require CFD experts. Computer science can automate a good portion of the CFD simulation processes, thus saving much human time required to obtain solutions. However, blind application of tools without understanding capabilities and limitations of the methods involved could lead to catastrophic engineering results. As in many other engineering and science disciplines, CFD researchers and practitioners need to understand the physics and engineering systems being simulated. Future experts will need to be cultivated who are willing to think through the flow physics in addition to the software engineering.

\section{REFERENCES}

1. Quarteroni, A., "Modeling the Cardiovascular System: A Mathematical Adventure -Part I," SIAM News, Vol. 34, No. 5, 2000.

2. Thom, A., "The Flow Past Circular Cylinder at Low Speeds," Proceedures, Royal Society Of London, Series A, Vol. 141, 1933, pp 651-666.

3. Roach, P.J., Computational Fluid Dynamics, Hermosa Publishers, Albuquerque, New Mexico, 1972.

4. Peyret, R. and Taylor, T.D., Computational Methods for Fluid Flow, Springer Series in Computational Physics, Springer-Verlag, 1983.

5. Hirsch, C., Numerical Computation of Internal and External Flows, John Wiley \& Sons, 1989.

6. Kwak, D., "Computation of Viscous Incompressible Flows," von Karman Institute for Fluid Dynamics, Lecture Series 1989-04[94?2004?]. Also NASA TM 101090, March 1989.

7. Gunzburger, M.D. and Nicolades, R. A., Incompressible Computational Fluid Dynamics Trends and Advances, ed. Cambridge University Press, 1993.

8. Hafez, M. and Oshima, K., Computational Fluid Dynamics Review 1998, World Scientific, 1998.

9. Gresho, P. M., and Sani, R. L., Incompressible flow and the finite element method, John Wiley and Sons, April 1998.

10. Hafez, M., Numerical Simulation of Incompressible Flows, World Scientific, 2002.

11. Harlow, F. H. and Welch, J. E., "Numerical Calculation of Time-Dependent Viscous Incompressible Flow with Free Surface," Phys. Fluids, Vol. 8, no. 12, pp. 2182-2189, Dec. 1965.

12. Gresho, M. P. and Sani, R. L., "On Pressure Boundary Conditions for the Incompressible Navier-Stokes Equations," Interntaional Journal of Numerical Methods in Fluids, Vol. 7, pp. 1111-1145, 1987:

13. Caretto, L.S., Gosman, A.D., Patankar, S.V. and Spalding, D.B., "Two Calculation Procedures for Steady Three-Dimensional Flows with Recirculation," Proceedings of the 3rd International Conference on Numerical Methods in Fluid Dynamics, Paris, France, p. 60, 1972.

14. Patankar, S.V., "Numerical heat transfer and fluid flow," Hemisphere Publishing Co., New York, 1980.

15. Chen, Y. S., Shang, H. M., Chen, C. P., "Unified CFD Algorithm with a Pressure Based Method," $6^{\text {th }}$ International Symposium on CFD, Sept. 4-8, 1995, Lake Tahoe, NV.

16. Chorin, A.J., "Numerical solution of Navier-Stokes equations," Mathematics of Computation, vol. 22, no. 104, 745-762, 1968.

17. Yanenko, N.N., The Method of Fractional Steps, Springer-Verlag, Berlin, 1971. 
18. Marchuk, G.M., Methods of Numerical Mathematics, Springer-Verlag, 1975.

19. Temam, R., Navier Stokes Equations, Revised Edition, North Holland, 1979.

20. Dwyer, H.S., Soliman, M. and Hafez, M., "Time accurate solutions of the Navier-Stokes equations for reacting flows," Proceedings of the $10^{\text {th }}$ Interntional Conference on Numerical Methods in Fluid Dynamics, Beijing, China, pp. 247-251, Springer-Verlag, June 1986.

21. Orszag, S. A., Israeli, M. and Deville, M.O., "Boundary Conditions for Incompressible Flows," Journal of Scientific Computing, Vol. 1, pp. 75-111, 1986.

22. Rosenfeld, M., Kwak, D., and Vinokur, M., "A Fractional-step Method for Unsteady Incompressible Navier-Stokes Equations in Generalized Coordinate systems," Journal of Computational Physics, Vol. 94, No. 1, May 1991, pp. 102-137.

23. Kiris, C. and Kwak, D., "Numerical Solution of Incompressible Navier-Stokes Equations Using a Fractional-step Approach," Computers and Fluids, 30, 2001, pp. 829-851.

24. Loner, R., Yang, C., Cebra!, J., Soto, O., and Camelli, F., "On Incompressible Flow Solvers," Numerical Simulation of Incompressible Flows, World Scientific, 2002.

25. Chorin, A. J., "A Numerical Method for Solving Incompressible Viscous Flow Problems," Journal of Computatinal Physics, Vol. 2, pp.12-26, 1967.

26. Chang, J. L. C., and Kwak, D., "On the Method of Pseudo Compressibility for Numerically Solving Incompressible Flows," AIAA Paper 84-0252, 1984.

27. Kwak, D., Chang, J. L. C., Shanks, S. P., and Chakravarthy, S., "A Three-Dimensional Incompressible Navier-Stokes Flow Solver Using Primitive Variables," AIAA Journal, Vol. 24, no. 3, 390-396, Mar. 1986.

28. Chang, J.L.C., Kwak, D., Rogers, S. E. and Yang, R-J, "Numerical Simulation Methods of Incompressible Flows and an Application to the Space Shuttle Maine Engine," International Journal of Numerical Method in Fluids, Vol. 8, pp. 1241-1268, 1988.

29. Choi, D. and Merkle, C. L., "Application of Time-iterative Schemes to Incompressible Flow," AIAA J., Vol. 23, no. 10, 1518-1524, 1985 .

30. Merkle, C. L. and Athavale, M., "Time-Accurate Unsteady Incompressible Flow Algorithms Based on Artificial Compressibility," AIAA Paper 87-1137, 1987.

31. Rogers, S. E., Kwak, D. and Kiris, C., "Steady and Unsteady Solutions of the Incompressible Navier-Stokes Equations," AIAA Journal Vol. 29, No. 4, 603-610, April 1991.

32. Turkel, E., 'Preconditioning Techniques in Computational Fluid Dynamics,' Annual Review of Fluid Mechanics, Vol. 31, 1999

33. Van Leer, B., Lee, W. T., and Roe, P. L., "Characteristic Time-Stepping or Local Preconditioning of the Euler Equations," AIAA Paper 91-1552-CP, Computational Fluid Dynamics Conference, Honolulu, HI, 1991.

34. Choi, Y-H, and Merkle, C. L., "The Application of Preconditioning in Viscous Flows," Journal of Computational Physics, Vol. 105, 1993, pp. 207-223.

35. Venkataswaran, S., and Merkle, C. L., "Evolution of Artificial Compressibility Methods in CFD," Numerical Simulation of Incompressible Flows, World Scientific, 2002.

36. Arabshahi, A., Taylor, L. K, and Whitfield, D. L., "UNCLE: Toward a Comprehensive Time-Accurate Incompressible Navier-Stokes Flow Solver," AIAA Paper No. 95-0050, AIAA 33rd Aerospace Sciences Meeting and Exhibit, Reno, NV, January 9-12, 1995.

37. Beam, R. M., and Warming, R. F., "An Implicit Factored Scheme for the Compressible Navier-Stokes Equations," AIAA Journal, Vol. 16, pp. 393-402, 1978.

38. Briley, W.R. and McDonald, H., "Solution of the Multidimensional Compressible Navier-Stokes Equations by a Generalized Implicit Method," Journal of Computational Physics, Vol. 24, No. 4, 1977, pp. 372-397.

39. Pulliam, T. H., and Chaussee, D. S., "A Diagonal Form of an Implicit Approximate-Factorization Algorithm," Journal of Computational Physics, Vol. 39, pp. 347-363, 1981.

40. Roe, P. L., "Approximate Riemann Solvers, Parameter Vectors, and Difference Schemes," Journal of Computational Physics, Vol. 43, p. 357, 1981.

41. Chakravarthy, S. R., and Osher, S., "A New Class of High Accuracy TVD Schemes For Hyperbolic Conservation Laws," AIAA Paper 85-0363, 1985.

42. Steger, J. L., and Warming, R. F., "Flux Vector Splitting of the Inviscid Gasdynamic Equations With Application to Finite Difference Methods," Joumal of Comp. Physics, Vol. 40, No. 2, pp. 263-293, 1981.

43. Harten, A., Lax, P. D., and Van Leer, B., "On Upstream Differencing and Godunov-Type Schemes for Hyperbolic Conservation Laws," Siam Review, Vol. 25, no. 1, p. 35, 1983.

44. MacCormack, R. W., "Current Status of Numerical Solutions of the Navier-Stokes Equations," ALAA Paper 85-0032, 1985. 
45. Glowinski, R., Juarez, H., and Pan, T-W, “ On the Numerical Simulation of Incompressible viscous Fluid Flow around Moving rigid Bodies of Elliptical Shapes," Numerical simulations of Incompressible Flows, World Scientific, 2002.

46. Morgan, K, Harlan, D., Hassan, O. Sorensen, K., and Weatherill, N., "Steady Incompressible Inviscid and viscous Flow simulation Using Unstructured Tetrahedral Meshes," Numerical Simulation of Incompressible Flows, World Scientific, 2002.

47. Tezduyar, T. E., "Stabilized finite element Formulations and Interface-Tracking of Interface-Capturing Techniques for Incompressible Flows," Numerical Simulation of Incompressible Flows, World Scientific, 2002.

48. Kiris, C., Kwak, D., and Rogers, S., "Incompressible Navier-Stokes Solvers in Primitive Variables and their Applications to Steady and Unsteady Flow Simulations," Numerical Simulation of Incompressible Flows, World Scientific, 2002.

49. Yoshida, Y., and Nomura, T., "A Transient Solution Method for the Finite Element Incompressible NavierStokes Equations," International .Tournal of Numerical Methods in Fluid, Vol. 5, pp. 873-890, 1985.

50. Taneda, S. and Honji, H., "Unsteady Flow Past a Flat Plate Normal to the Direction of Motion," Journal of Phys. Society, Japan, Vol. 30, pp. 262-273, 1971.

51. Steger, J. L., Dougherty, F. C., and Benek, J. A., 'A Chimera Grid Scheme,' Advances in Grid Generation, K. N. Ghia and U. Ghia, eds. ASME FED-Vol. 5, June, 1983.

52. Kiris, C., Chan, W., and Kwak, D., "A Three-Dimensional Parallel Time-Accurate Turbopump Simulation Procedure Using Overset Grid System," Second International Conference on Computational Fluid Dynamics, Sydney, July 15-19, 2002.

53. Womersley J. R., "An Elastic Tube Theory of Pulse Transmission and Oscillatory Flow in Mammalian Arteries," Wright-Patterson Air Force Base, OH, 1957.

54. Berger, S. A. and Jou, L-D., "Flow in Stenotic Vessels," Annual Review., Fluid Mech. 32:347-382, 2000.

55. Quarteroni, A., "Modeling the Cardiovascular System: A Mathematical Challenge," Mathematics Unlimited-2001 and Beyond, Enquist \& Schmid (eds), October 9, 2001.

56. Fernandez, A., David, T. and Brown, M. D., "Numerical Models of Auto-regulation and blood Flow in the Cerebral Circulation," Computer Methods in Biomechanics and Engineering, Vol., 5 (1), pp. 7-20, 2002.

57. Kim, C.S., Kiris, C., Kwak, D., "Numerical Models of Human Circulatory System under Altered Gravity: Brain circulation," AIAA Paper No. 2004-1092, AIAA 42nd Aerospace Sciences Meeting and Exhibit, Reno, NV, January 5-8, 2004.

58. Smith, N. P., Pullan, A. J., and Hunter, P. J., "An Anatomically Based Model of Transient Coronary Blood Flow in the Heart," SIAM Journal of Applied Mathetmatics, Vol. 62, No. 3, pp. 990-1018, 2002.

59. Peskin, C. S., McQueen, M., "Computational Biofluid Dynamics," Fluid Dynamics in Biology, Seattle WA, American Math Society, pp. 161-186, 1993.

60. Kiris, C., Kwak, D., and Benkowski, R., "Incompressible Navier-Stokes Calculations for the Development of a Ventricular Assist Device," Computer and Fluids, Vol. 27, Nos. 5-6, pp. 709-719, 1998. 